\title{
Pulmonary Embolism: Pathophysiologic Insights for New Treatment Trials
}

\author{
Editorial to: "Doxycycline Prevents Acute Pulmonary Embolism-Induced Mortality \\ and Right Ventricular Deformation in Rats" by Stefany B.A. Cau et al.
}

\author{
Kenneth E. Wood
}

Published online: 15 May 2013

(C) Springer Science+Business Media New York 2013

Despite dramatic advances in prophylactic and diagnostic modalities for pulmonary embolism (PE), treatment has not meaningfully changed over the past three decades; hemodynamically stable patients receive heparin and hemodynamically unstable patients are recommended to undergo medical embolectomy with thrombolytic therapy or when contraindicated, catheter or surgical embolectomy. Albeit the mortality for hemodynamically stable patients is low, the mortality for patients with either right ventricular dysfunction (RVD) or hemodynamic instability remains unacceptably high, necessitating a search for new innovative treatment strategies. In this issue of Cardiovascular Drugs and Therapy, Cau et al. present data and a cogent argument for initiating clinical trials of novel treatment modalities for PE [1].

Therapeutic advances are often predicated upon similar advances in the understanding of disease state pathophysiology. RVD has traditionally been attributed to the combination of acute PE induced pressure overload, which is a function of both mechanical obstruction and vasoconstrictive forces in combination with RV ischemia. The latter occurs consequent to PE induced increases in RV wall stress and a loss of the RV coronary perfusion pressure gradient which occurs when mean arterial pressure decreases and RV end-diastolic pressure increases in hemodynamically unstable patients. Recent reports suggest that the PE induced pressure load produces sacromere stretch resulting in an RV inflammatory state that further jeopardizes RV function [2]. Human autopsy series of fatal PE cases with large clot

K. E. Wood $(\bowtie)$

Geisinger Medical Center, 100 N Academy Avenue,

Danville, PA 17822, USA

e-mail: kewood@geisinger.edu burden have identified necroinflammatory changes in histologic sections of the RV myocardium. Inflammatory infiltrate consisting of macrophages, T-cells and neutrophils with myocyte necrosis was recognized in association with the preceding inflammatory foci in $64 \%$ and a 6.1 fold increase in RV fibrosis was noted [3, 4].

In animal models, RV myocardial neutrophilic infiltration is reported to contribute to RV damage and occurs early (6-18 h) after the event [5], whereas macrophage infiltration persists and is biphasic. Initially macrophages with an MI phenotype predominate (inflammatory), whereas those with an M2 phenotype (healing) are prominent at 6 weeks [6]. Similar finding of a neutrophil and macrophage mediated inflammatory response with accompanying intimal hyperplasia have been reported in the pulmonary artery after PE [7]. Attenuation of the inflammatory response and prevention of RV dysfunction with anti-neutrophilic antibodies and inhibition of chemokine CINC-1 (cytokine-induced neutrophil chemoattractant) further support the role of neutrophil induced inflammation as a culprit for RV dysfunction [5, 7].

The preceding RV damage appears to be mediated by the up-regulation of matrix metalloproteinases (MMPs) [8-11] which are endopeptidases localized to the sarcomere within the cardiocyte and are engaged in the normal turnover of components of the extracellular matrix [12]. Cardiac MMPs are activated by neutrophil generated oxidative stress [12] or neutrophils may release MMP granules [13] which have been shown to degrade sarcomeric and cytoskeletal proteins along with troponin I and myosin light chains causing myocardial dysfunction [10]. Serum troponin I and plasma MMP concentrations have been reported to correlate with pulmonary vascular resistance and pulmonary artery pressure in acute PE [10]. MMPs derived from neutrophils reportedly can degrade endothelin-1, forming a more potent 
vasoconstrictor which can further increase pulmonary vascular resistance [14]. Inhibition of MMPs by doxycycline has been reported to attenuate PE hemodynamic changes [8] and blunt troponin increases [8, 9, 11].

In this issue, Cau et al. have advanced the preceding experimental literature by reporting that pharmacologic inhibition of MMPs by doxycycline decreased RV dilatation and mortality in an animal model of PE. Similar hemodynamic attenuation and mortality benefits were seen when atorvastatin was used to inhibit MMPs in experimental animal models [15]. After three decades of static therapy for acute PE, advances in the pathophysiologic understanding of the disease mandate that novel physiologically based therapies begin human trials in this lethal disease.

\section{References}

1. Cau SBA, Barato RRC, Celes MR, Muniz JJ, Rossi MA, TanusSantos JE (2013) Doxycycline prevents acute pulmonary embolism-induced mortality and right ventricular deformation in rats. Cardiovasc Drugs Ther 2013;27:this issue

2. Watts JA, Marchick MR, Kline JA. Right ventricular heart failure from pulmonary embolism - key distinctions from chronic pulmonary hypertension. J Cardiac Fail. 2010;613:250-9.

3. Orde MM, Puranik R, Morrow PL, Duflou J. Myocardial pathology in pulmonary thromboembolism. Heart. 2011;97:1695-9.

4. Begieneman MPV, van de Goot FRW, van de Bilt IAL, et al. Pulmonary embolism causes endomyocarditis in the human heart. Heart. 2008;94:450-6.
5. Watts JA, Zagorski J, Gellar MA, Stevinson BG, Kline JA. Cardiac inflammation contributes to right ventricular dysfunction following experimental pulmonary embolism in rats. J Mol Cell Cardiol. 2006;41:296-307.

6. Watts JA, Gellar MA, Obraztsova M, Kline JA, Zagorski J. Role of inflammation in right ventricular damage and repair following experimental pulmonary embolism in rats. Int $\mathrm{J}$ Exp Path. 2008;89:389-99.

7. Zagorski J, Gellar MA, Obraztsova A, et al. Inhibition of CiNC-1 decreases right ventricular damage caused by experimental pulmonary embolism in rats. J Immunol. 2007;179:7820-6.

8. Palei AC, Zaneti RAG, Fortuna GM, et al. Hemodynamic benefits of matrix metalloproteinase-9 inhibition by doxycycline during experimental pulmonary embolism. Angiology. 2005;56:611-7.

9. Fortuna GM, Figuerido-Lopes L, Dias-Junior CAC, et al. A role for matrix metalloproteinase-9 in hemocynamic changes following acute pulmonary embolism. Int J Cardiol. 2007;114:22-7.

10. Uzuelli JA, Diaz-Junior CA, Tanus-Santos JE. Severity of dependent increases in circulating cardiac troponin-I and MMP-9 concentrations after experimental acute pulmonary thromboembolism. Clin Chim Acta. 2008;388:184-8.

11. Neto-Neves E, Diaz-Junior CA, Rizzi E. Metalloproteinase inhibition protects against cardiomyocyte injury during experimental acute pulmonary embolism. Crit Care Med. 2011;39:349-56.

12. Schultz R. Intracellular targets of matrix metalloproteinase-2 in cardiac disease: rational and therapeutic approaches. Ann Rev Pharmacol Toxicol. 2007;47:211-42.

13. Van den Steen PE, Dubois B, Nelissen P, et al. Biochemistry and molecular biology of gelatinase B or matrix metalloproteinase- 9 (MMP-9). Crit Rev Biochem Mol Biol. 2002;37:375-536.

14. Fernandez-Patron C, Radomski MW, Davidge ST. Vascular matrix metalloproteinase-2 cleaves big endothelin-1 yielding a novel vasoconstrictor. Circ Res. 1999;85:906-11.

15. Souza-Costa DC, Figuierdo-Lopes L, Alves-Filbo JL, et al. Protective mechanisms of atorvastin in rat models of acute pulmonary embolism: involvement of matrix metalloproteinase-9. Crit Care Med. 2007;35:239-45. 